\title{
Rationale and Design of the TARGET-EFT Trial: Multicomponent Intervention for Frail and Pre-frail Patients Hospitalized with Acute Cardiac Conditions
}

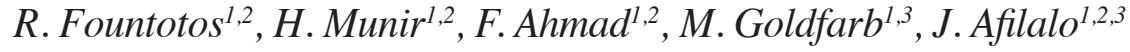

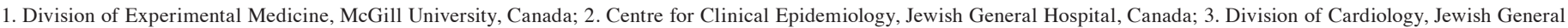 \\ Hospital, McGill University, Canada

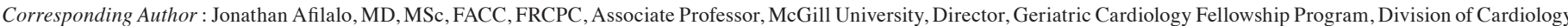

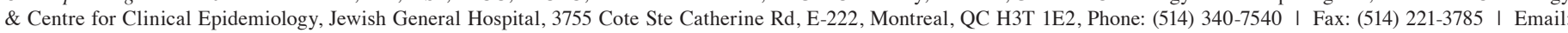
jonathan.afilalo@mcgill.ca

\begin{abstract}
BACKGROUND: With the aging population and rising rates of cardiovascular disease (CVD), cardiologists and cardiac surgeons are encountering a growing number of frail older patients that have complex cardiac and non-cardiac issues. Measuring frailty provides valuable prognostic information to help personalize treatment decisions. However, there is minimal evidence on multicomponent frailty interventions in this setting. The TARGET-EFT (The MulTicomponent Acute Intervention in FRail GEriatric PaTients with cardiovascular disease using the Essential Frailty Toolset) trial aims to target physical and non-physical frailty deficits to improve healthrelated quality of life and hospital-acquired disability in frail patients hospitalized with CVD.

METHODS: The TARGET-EFT trial is a single-center parallelgroup randomized clinical trial in frail and pre-frail older adults $\geq 65$ years admitted to the cardiovascular unit (CVU) at the Jewish General Hospital, Montreal, Quebec. The trial will compare usual inpatient care to a multicomponent intervention targeting physical weakness, cognitive impairment, malnutrition, and anemia. Outcomes of interest in both groups will be assessed at three time points: (1) study enrollment, (2) discharge from the CVU, and (3) 30 days after hospital discharge.

CONCLUSIONS: The overarching goal is to treat patients' frailty in parallel with their CVD, and in doing so, optimize patient functional losses while in-hospital and shortly thereafter. The results of this trial will inform best practices for patient-centered care in this vulnerable patient group.
\end{abstract}

Key words: Frailty, intervention, cardiovascular disease.

\section{Introduction}

$\mathrm{F}$ Trailty is a multidimensional geriatric syndrome, characterized by an increased vulnerability to stressors, observed in $20-60 \%$ of patients with cardiovascular disease (CVD) (1). Frailty is a major risk factor for adverse events in cardiac patients, including protracted recovery, functional decline, poor health-related quality of life (HRQOL) and death (1). With today's aging population and rising rates of CVD, cardiologists and cardiac surgeons are encountering a growing number of frail older patients that have complex cardiac and non-cardiac issues. Measuring frailty in clinical practice provides valuable prognostic information to help personalize treatment decisions (2). The Essential Frailty Toolset (EFT) is one of several tools that has been validated to predict death and functional decline in older patients undergoing cardiac surgery and transcatheter aortic valve replacement $(3,4)$. In addition to being among the most predictive tools and easiest-to-administer, the EFT was conceived to be actionable via practical interventions aimed at correcting the elicited deficits (5).

During the course of a hospital admission for CVD, frailty can be exacerbated by both illness-related and hospitalizationrelated stressors such as sleep deprivation, undernutrition, pain, anxiety, polypharmacy, and immobility (6). These stressors exceed the frail patient's homeostatic reserves and thus lead to decompensation in the form of delirium, functional decline, complications, and readmissions; which has been termed "posthospital syndrome" $(6,7)$. The ensuing physical deconditioning can also precipitate disabilities in activities of daily living (ADLs), known as hospital-acquired disability (HAD) (8, 9). It has been reported that $50 \%$ of hospitalized patients over the age of 85 years acquire at least one new disability in ADLs upon discharge (8), placing them at risk for loss of functional independence and institutionalization. Among patients who developed HAD, $41 \%$ died within the first year post-hospitalization and $29 \%$ retained an acquired disability beyond one year (10).

Therapeutic de-frailing interventions (such as inpatient mobilization, nutritional supplementation, cognitive stimulation and anemia correction) can mitigate the effect of hospitalization-related stressors. Consequently, these interventions can help prevent iatrogenic complications and deconditioning. Ultimately, these contribute to avoiding HAD and improving patients' HRQOL. There is minimal evidence on targeted multicomponent interventions addressing frailty in geriatric patients within acute cardiac care settings (11). The MulTicomponent Acute Intervention in FRail GEriatric PaTients with cardiovascular disease using the Essential Frailty Toolset (TARGET-EFT) randomized clinical trial aims to address this knowledge gap in patients with CVD. Through a patient-centered approach that focuses on physical activity, nutrition, and delirium prevention, this trial aims to improve HRQOL and prevent HAD in frail older patients hospitalized with CVD. 
The primary objectives of the trial are:

1. To determine whether a multicomponent geriatric intervention to improve frailty, as measured with the EFT, will improve short-term HRQOL at 30 days post-discharge.

2. To determine whether this multicomponent geriatric intervention will reduce HAD at 30 days post-discharge.

The secondary objectives of the trial are to determine whether the intervention will reduce 30-day readmission or death, improve physical performance and frailty status, and reduce in-hospital anxiety and depression.

\section{Figure 1. Essential Frailty Toolset (EFT)}

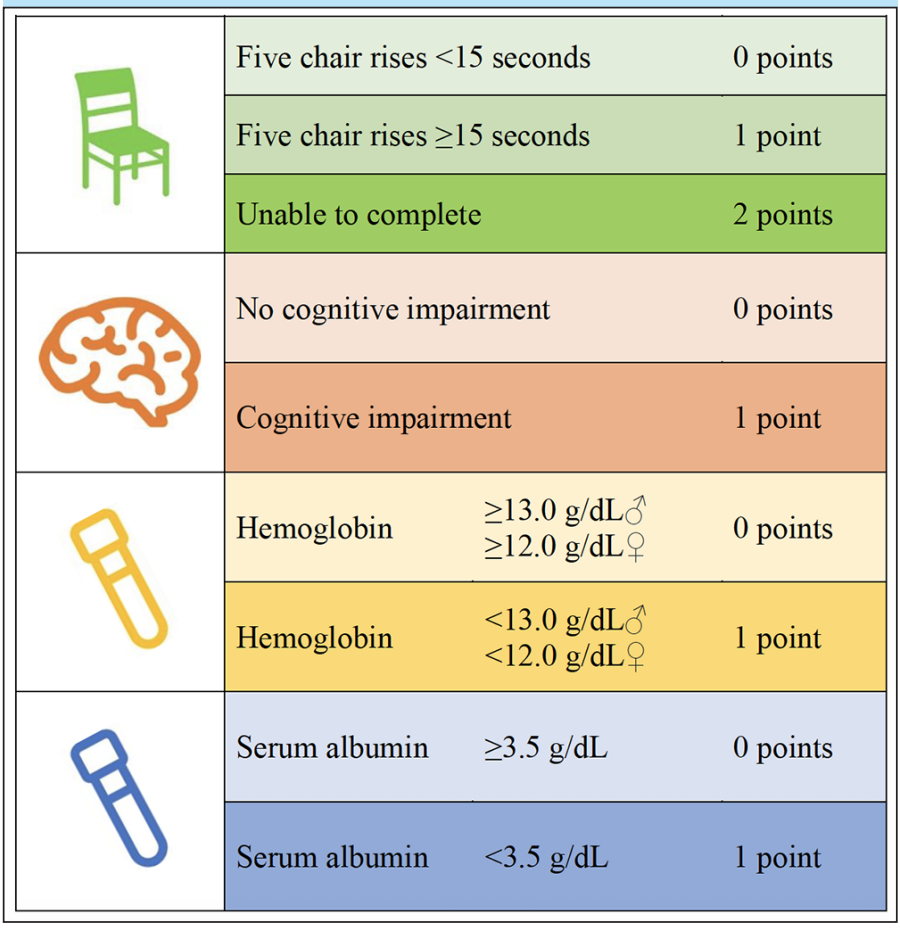

The EFT is scored 0 to 5 based on the following four assessments at the patient's bedside: five chair rises (without using arms), three-word recall and orientation to time and place, and verification of their recent hemoglobin and serum albumin values. EFT scores of 1-2 are considered pre-frail, while scores of 3-5 are considered frail.

\section{Methods}

\section{Study Design}

The TARGET-EFT trial is a single-center parallel-group randomized clinical trial that will compare usual inpatient care to a multicomponent geriatric intervention in frail and pre-frail older adults admitted to the cardiovascular unit (CVU) at the Jewish General Hospital, McGill University, Montreal, Quebec. The CVU is an integrated practice unit with cardiology and cardiac surgery patients, staffed by a multidisciplinary team including: cardiologists, cardiac surgeons, nurse practitioners, nurses, residents, medical students, occupational therapists, nutritionists, and physiotherapists. The unit has 45 beds and admits 2,200 patients per annum.

The target population encompasses acute cardiac conditions such as acute coronary syndromes, acute decompensated heart failure, arrythmias, recent cardiac surgery, symptomatic valvular heart disease, myopericarditis and pulmonary hypertension. The trial will recruit 144 patients (72 per group). Patients who meet the inclusion criteria and consent to participate will be randomly allocated to the control group consisting of usual clinical care, or the intervention group consisting of treatments targeted to the EFT deficits identified. Outcomes of interest in both groups will be assessed at three time points: (1) study enrollment, (2) discharge from the CVU, and (3) 30 days after hospital discharge. The study flowchart is illustrated in Figure 2. The conceptual diagram is illustrated in Figure 3, showing how the TARGET-EFT interventions aim to counteract frailty deficits and hospitalization-related stressors.

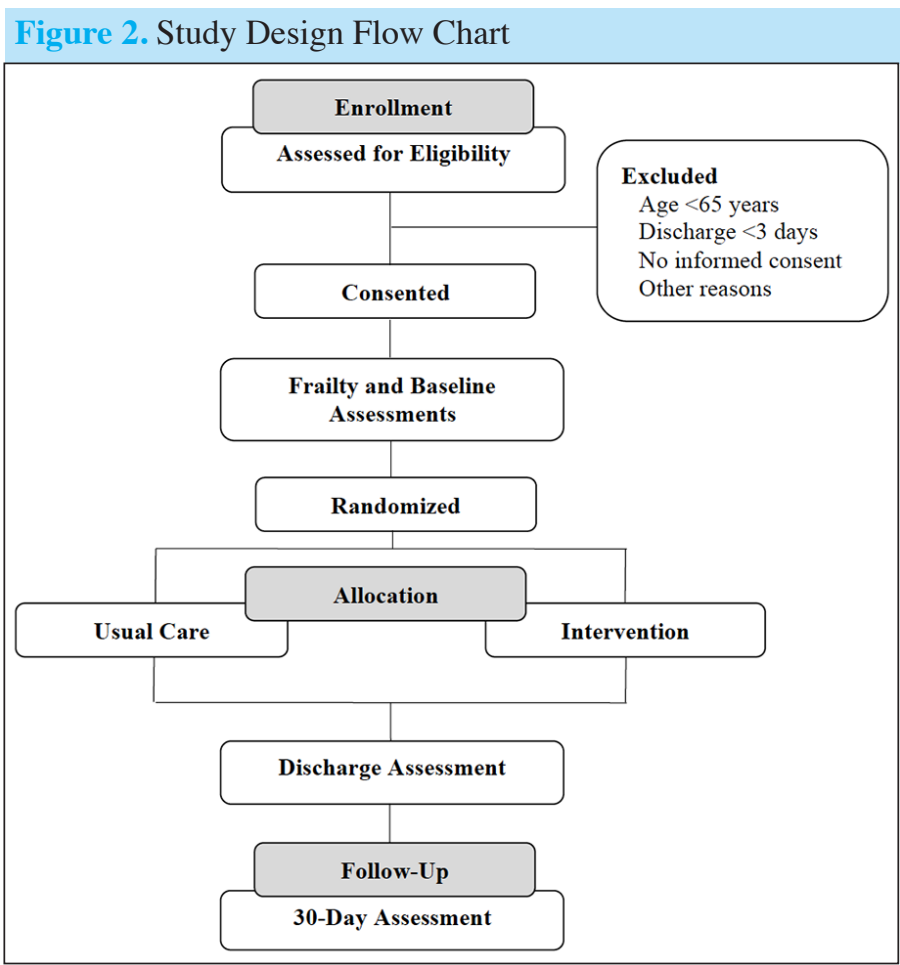

Patients admitted to the cardiovascular unit will be screened for eligibility based on their EFT score, age, and anticipated discharge date. Those who consent will undergo baseline assessments and be randomized to the intervention or usual care group, which they will receive throughout their hospital stay. Abbreviations: EFT, Essential Frailty Toolset; HADS, Hospital Anxiety and Depression Scale; OARS, Older Americans Resources and Services; SPPB, Short Physical Performance Battery.

The trial is approved by the Research Ethics Board of the Jewish General Hospital and registered on the National Institutes of Health's clinical trials database ClinicalTrials. gov (NCT04291690). Graduate students funded by research scholarships (see Funding section) will coordinate all aspects of the trial in collaboration with the treating cardiology teams.

\section{Participant Selection}

\section{Inclusion criteria}

\section{Age $\geq 65$ years}

2. Frail or pre-frail, defined by an EFT score $\geq 1$

3. Admitted to the CVU

4. Provided written informed consent 


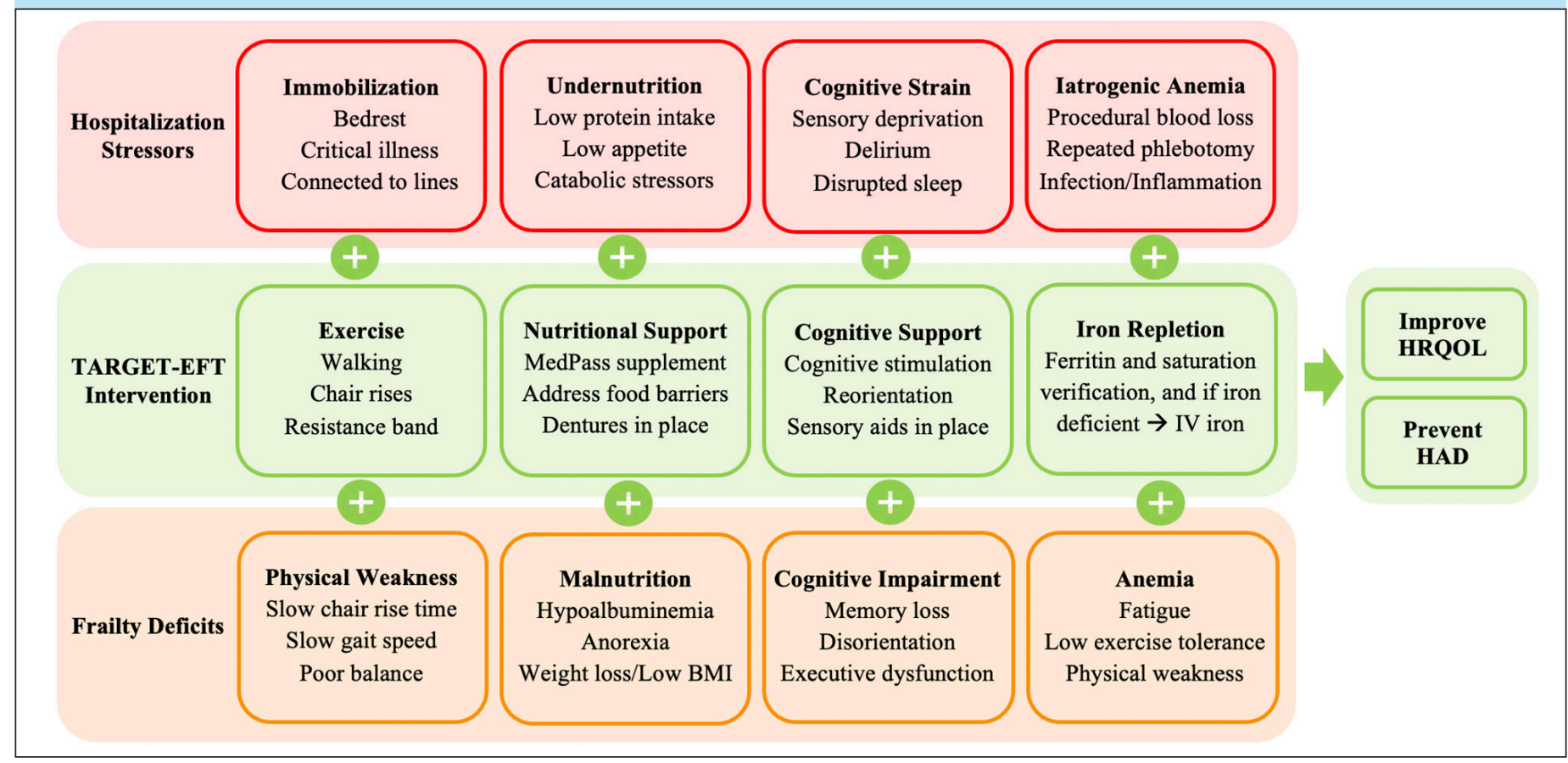

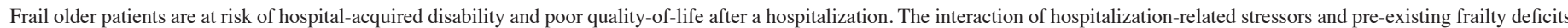

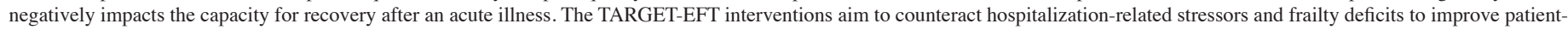
centered outcomes. Abbreviations: BMI, body mass index; HAD, hospital-acquired disability; HRQOL, health-related quality of life; IDA, iron deficiency anemia; IV, intravenous.

\section{Exclusion criteria}

1. Expected discharge within $<3$ days

2. Clinically unstable, defined as any of:

a. Unstable vital signs

b. Low-threshold coronary ischemia

c. Uncontrolled decompensated heart failure

d. Uncontrolled arrhythmia

3. Awaiting cardiac surgery within $<3$ days

4. Severe cognitive impairment, defined by a score of $\leq 10 / 30$ on the Mini-Mental Status Examination (MMSE)

5. Active delirium, defined by the Confusion Assessment Method (CAM)

6. Psychiatric conditions precluding cooperation

7. Parkinson's disease

8. Recent stroke within $<7$ days

9. Marked physical limitations or bed-bound precluding participation in exercise interventions

10. End-of-life care plan

11. COVID-19 positive or not yet ruled out

12. Not English or French speaking

\section{Screening Process}

Research personnel will liaise on weekdays and most weekends with the nursing staff and allied health professionals in the CVU to pre-screen patients admitted that day or within the last three days. Patients' care needs, level of mobility, clinical status, and discharge plan will be discussed with the treating team. Research personnel will then screen potentially eligible patients using the EFT, consisting of four components: physical weakness (measured by the time to complete five chair rises), cognitive impairment (measured by a three-word recall and orientation to time and place), malnutrition (measured by serum albumin), and anemia (measured by serum hemoglobin). An EFT score of $\geq 1-2$ is indicative of pre-frailty and a score of 3-5 is indicative of frailty (Figure 1). Patients who meet these criteria and provide written informed consent to participate will be randomly allocated to one of two parallel groups and undergo an in-depth frailty assessment.

\section{Frailty and Baseline Assessments}

The frailty and the baseline assessments consist of an interviewer-administered questionnaire, anthropomorphic measurements, and physical performance tests conducted by research personnel. In addition to the EFT, the following measures will be captured: Short Performance Physical Battery (SPPB), Preoperative Nutrition Score (PONS), MMSE and hemoglobin (12-14). The SPPB is composite score from 0-12 consisting of time to complete five chair rises, time to walk five meters, and ability to stand for 10 seconds in side-by-side, semi-tandem, and tandem positions. The PONS is a composite score from 0-4 consisting of low body mass index, weight loss, low appetite, and low albumin level. The following covariates will be recorded: age, sex, height, weight, reason for hospital admission, comorbidities, cardiac investigations, and treatments received.

\section{Randomization and Blinding}

Patients will be randomly allocated to either the intervention or control group on a 1:1 basis in concealed blocks of eight 
Table 1. Usual Care and TARGET-EFT Intervention

\begin{tabular}{|c|c|c|c|c|}
\hline & Physical Weakness & Cognitive Impairment & Malnutrition & Anemia (Iron-Deficient) \\
\hline Usual care & $\begin{array}{l}\text { - RN encouragement to mobilize } \\
\text { early and often } \\
\text { - Physiotherapy PRN }\end{array}$ & $\begin{array}{l}\text { - RN orientation } \\
\text { - RN screening for delirium } \\
\text { - Geriatrics consult PRN }\end{array}$ & $\begin{array}{l}\text { - RN encouragement to eat } \\
\text { hospital meals } \\
\text { - Nutritionist screening and } \\
\text { intervention PRN } \\
\text { - MedPass PRN }\end{array}$ & $\begin{array}{l}\text { - MD investigation for diagnosis } \\
\text { of anemia } \\
\text { - MD prescription for oral or } \\
\text { intravenous iron replacement } \\
\text { PRN }\end{array}$ \\
\hline Intervention & $\begin{array}{l}\text { - Additional encouragement to } \\
\text { mobilize between and during visits } \\
\text { BID } \\
\text { - Chair rises at least BID } \\
\text { - If SPPB } \leq \mathbf{9} \text { : Vivifrail program } \\
\text { x BID }\end{array}$ & $\begin{array}{l}\text { - Put on hearing/vision aids } \\
\text { - Additional orientation on board } \\
\text { and during visits BID } \\
\text { - Address sleep promotion } \\
\text { - If MMSE } \leq \mathbf{2 6 / 3 0} \text { : cognitive } \\
\text { stimulation BID }\end{array}$ & $\begin{array}{l}\text { - Additional encouragement to } \\
\text { eat during visits BID } \\
\text { - Address food likes/barriers } \\
\text { - If PONS +: prescription of } \\
\text { MedPass QID }\end{array}$ & $\begin{array}{l}\text { - Put on dentures } \\
\text { - Verification of iron studies } \\
\text { according to established } \\
\text { diagnostic criteria } \\
\text { - If iron deficient anemia: } \\
\text { prescription of intravenous iron } \\
\text { QD x } 3\end{array}$ \\
\hline
\end{tabular}

Patients allocated to the intervention group will receive usual clinical care plus the multicomponent trial intervention, whereas those allocated to the control group will receive usual clinical

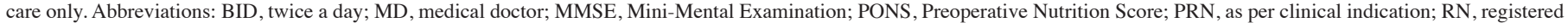
nurse; SPPB, Short Physical Performance Battery; QD, once a day; QID, four times a day.

Table 2. Clinical Outcomes of Interest and Ascertainment Time Points

\begin{tabular}{|c|c|c|c|c|}
\hline & Measured by & Baseline & Discharge & 30 Days \\
\hline \multicolumn{5}{|l|}{ Primary Outcome } \\
\hline Health-related quality of life & EQ-5D-5L & $\mathrm{X}$ & $\mathrm{X}$ & $\mathrm{X}$ \\
\hline Hospital-acquired disability & OARS & $\mathrm{X}$ & $\mathrm{X}$ & $\mathrm{X}$ \\
\hline Length of stay & Days & & $\mathrm{X}$ & \\
\hline Lower extremity physical performance & SPPB & $\mathrm{X}$ & $\mathrm{X}$ & \\
\hline All-cause death, discharge to healthcare facility, unplanned repeat hospital visit & Composite benefit & & & $\mathrm{X}$ \\
\hline
\end{tabular}

Abbreviations: OARS; Older Americans Resources and Services. Other abbreviations as in Table 1.

(block randomization) stratified by sex to ensure an equal distribution of males and females. Research personnel administering the intervention cannot feasibly be blinded to the allocation group, however, outcome ascertainers will be blinded; specifically, the primary outcome at discharge will be ascertained by a blinded clinical nurse, and the 30-day telephone questionnaires will be administered by a blinded member of the research team unaware of the allocation group so as to minimize bias (such as leading intervention patients towards more favorable responses on questionnaire-based outcome measures).

\section{Allocation Groups}

\section{Control group}

Patients randomized to the control group will receive usual clinical care as prescribed by their treating physicians. In addition to cardiovascular treatments, usual care may or may not include inpatient physiotherapy, nutritional consultation and supplementation, geriatric and psychiatric consultation, and treatment of anemia. Available resources do not allow for the involvement of physiotherapists, nutritionists, and geriatricians in all older patients, typically being reserved for those with apparent deficiencies seen by the treating physicians or nurses.

\section{Intervention group}

Patients randomized to the intervention group will receive an in-hospital multicomponent geriatric intervention to improve frailty deficits (“de-frailing"). The intervention will provide support for mobilization, orientation, and nutrition, as well as additional support targeted to patients' frailty deficits such as strength and balance exercise training for those with physical weakness, cognitive stimulation for those with cognitive impairment, oral nutritional supplementation for those with malnutrition risk, and intravenous iron replacement therapy for those with iron-deficiency anemia (Table 1).

Research personnel who will deliver the intervention are graduate students with accreditation in Kinesiology and experience providing exercise therapy to older adults. They will visit each patient twice-daily (once in the early morning and once in the afternoon) in their hospital room to provide the different components of the intervention. In cases of prolonged hospital stays, the frequency of visits will be reduced to once daily during the 3rd and 4th weeks of hospitalization, and once every other day thereafter. Visits may be deferred or rescheduled if patients are not feeling well, refuse, or are absent for scheduled tests during the planned visit time.

At the time of discharge, intervention patients will be encouraged to continue guideline-recommended physical and nutritional habits as instituted during their hospital stay (such as 150 minutes per week of physical activity, as feasible, and a balanced diet), with the support of their family members and caregivers and the oversight of their treating physician. Research personnel will not provide any interventional support or supervision after discharge. 


\section{Exercise component}

All intervention patients will be encouraged and assisted to walk around the ward, as tolerated and approved by their treating team, to maximize the time spent out of bed (at a minimum, up in chair for all meals). They will also be encouraged and assisted to perform the chair rise exercise to build lower extremity strength. The subset of intervention patients with greater physical weakness, defined as an SPPB score $\leq 9$, will be provided with a supervised exercise program combining strength, flexibility, balance, and mobility, with the repetitions and difficulty level tailored to their SPPB scores and physical capabilities. The exercise program is adapted from Vivifrail, a European Union-funded initiative to prevent frailty, physical deconditioning, and incident disability in older persons (Supplementary Table S1) (15). The exercise program is comprised of two daily sessions of 20 minutes each; the morning session will targeting lower-body strength with and flexibility, and the afternoon session will target balance and walking.

\section{$\underline{\text { Cognitive component }}$}

All intervention patients will be encouraged and assisted to wear hearing and visual aids (if applicable) and oriented to time and location, both verbally and in writing on a clearly visible whiteboard in their hospital room. Family members and caregivers will be encouraged to orient the patient as well. Researchers will inquire about sleep problems, which will be communicated to the treating team. The subset of intervention patients with greater cognitive impairments, defined as an MMSE score $\leq 26$, will be provided with cognitive stimulation comprising activities with the patient, including, but not limited to, current news, trivia, crossword puzzles, and memory games for a target of 20 minutes twice a day. The cognitive intervention is adapted from the Hospital Elder Life Program (HELP) to prevent delirium (16).

\section{$\underline{\text { Nutritional component }}$}

All intervention patients will be encouraged and assisted to wear their dentures (if applicable) and eat their hospital meals and snacks. Researchers will inquire about food preferences and eating barriers, which will be communicated to the dietician. The subset of intervention patients with greater nutritional deficits, defined as a positive PONS score, will be prescribed MedPass supplementation with Nestle Resource 2.0 or Ensure ProteinMax. MedPass consists of a $60 \mathrm{~mL}$, calorically dense, oral nutritional supplement prescribed by clinicians and delivered by nurses at the same time as medications (not at mealtime) four times per day. It has been shown to be effective in patients at risk of malnutrition and better tolerated in older adults as compared to protein supplements that are more voluminous and compete with mealtime food intake $(17,18)$. If MedPass supplementation is contraindicated, the treating team will have full discretion to avoid its prescription and consider alternative supplementation strategies.

\section{$\underline{\text { Anemia component }}$}

The subset of intervention patients with iron-deficiency anemia, defined as (i) hemoglobin $<130 \mathrm{~g} / \mathrm{L}$ in men or $<120$ $\mathrm{g} / \mathrm{L}$ in women will be assessed for low ferritin. Patients with (ii) ferritin $<100 \mu \mathrm{g} / \mathrm{L}$ or ferritin $<300 \mu \mathrm{g} / \mathrm{L}$ with a saturation $<20 \%$, will be prescribed intravenous iron replacement therapy by the treating physician. Specifically, three daily doses of iron sucrose $300 \mathrm{mg}$, as per the indication for intravenous iron replacement therapy based on the Canadian Cardiovascular Society Guidelines for Heart Failure (19). Intravenous mode of delivery is preferred because of its rapid effect as compared to oral supplementation, with hospitalization being an opportune moment to administer it (20). If intravenous iron therapy is contraindicated, the treating team will have full discretion to avoid its prescription and consider alternative strategies.

\section{Outcome Measures}

The primary outcome is HRQOL as measured by the EQ-5D$5 \mathrm{~L}$ scale at 30 days post-discharge. The EQ-5D-5L scale quantifies HRQOL in five dimensions of health: 1) mobility, 2) self-care, 3) usual activities, 4) pain/discomfort, and 5) anxiety/ depression. Each dimension has five response options ranging from no problems to severe problems, which are entered in a regression formula to compute a composite score ranging from 0 to 1 based on nationally adapted coefficients $(21,22)$. The EQ-5D-5L scale is self-reported and administered by an observer. For the TARGET-EFT trial, the observer will be the patient's treating nurse for the discharge assessment and a trained research assistant for the 30-day telephone assessment, both of which will be blinded to the patient's allocation group.

The main secondary outcome is hospital-acquired disability as measured by the Older Americans Resources and Services (OARS) scale at 30 days post-discharge (23). The OARS scale quantifies dependencies in seven basic and seven instrumental activities of daily living (ADLs, IADLs). Each activity has four response options to evaluate the capacity of the patient to complete them independently: without help, with some help, unable, and not applicable. The OARS scale is self-reported and administered by a trained research assistant blinded to the patient's allocation group at the 30-day telephone assessment.

Other secondary outcomes are physical frailty as measured by the Short Physical Performance Battery (SPPB) scale at discharge and the SARC-F questionnaire at 30 days postdischarge (24). Additional secondary outcomes are mood disturbance as measured by the Hospital Anxiety and Depression Scale (HADS) at discharge and length of stay from the day of randomization to hospital discharge (25). Table 2 lists the outcome measures.

\section{Statistical Analysis}

Data will be analyzed according to the intention-to-treat principle, modified to exclude patients who were censored pre-emptively before receiving a first visit or completing core assessments (due to early discharge or death). Multivariable linear regression will be used for continuous outcome measures 
(EQ-5D-5L, OARS, SPPB, SARC-F, HADS, length of stay) to determine the effect of the intervention after adjusting for baseline scale values and duration of the in-hospital intervention. Effect-modification with these and other relevant covariates will be examined. Missing data will be imputed using multiple imputation. Multivariable logistic regression will similarly be used for dichotomous composite outcome measures.

\section{Sample Size Calculation}

The sample size calculation is based on the primary outcome measure of HRQOL as measured by the EQ-5D-5L scale. A sample size of 72 patients per group $(\mathrm{N}=144)$ is required to detect a clinically meaningful change of $0.056 \pm 0.011$ points in the EQ-5D-5L scale given an alpha of 0.05 and a beta of 0.2 (26). This sample size accounts for an anticipated upper limit of $10 \%$ losses to follow-up, a conservative estimation given that the primary outcome is assessed shortly after discharge.

\section{Data Collection and Management}

Data will be collected on paper-based case report forms and entered in a REDCap database. REDCap is a web-based application for managing clinical study data, which is hosted on secure server located at the Jewish General Hospital's Lady Davis Institute for Medical Research. The patient's identification will be anonymized and codified using a 3-digit number followed by a 2-letter monogram; no identifying information will be disseminated in publications and scientific presentations.

\section{Ethics Approval}

The Jewish General Hospital's Research Ethics Board has approved this trial, which will be conducted in accordance with the Tri-Council Policy Statement on the Ethical Conduct for Research Involving Humans (2014) as well as the requirements set forth by the Research Ethics Board. Participation in the TARGET-EFT trial will be entirely voluntary, and patients will have the right to refuse or withdraw from the trial at any time. Regardless of a patient's enrolment in the trial, they will be entitled to receive mobilization, physiotherapy, nutritional supplementation, and anemia treatments as clinically indicated by the treating physicians and allied health professionals.

\section{Trial Monitoring}

Exercise-related symptoms and adverse events will be recorded and reviewed throughout the trial to ensure patient safety. Chest pain, dyspnea, palpitations, desaturation, lightheadedness, syncope, falls, musculoskeletal pain or discomfort, worsening heart failure or coronary ischemia will be considered as significant adverse events, and will be promptly reported to the Research Ethics Board for further review.

To minimize the risk of adverse events, heart rate and oxygen saturation will be monitored during exercise. Exercise intensity will be limited as a function of the patients' SPPB level, current health status, and perceived exertion using the 20-point Borg scale (27). The research team will consult with the clinical team on a daily basis to ensure that the patients are safe to undergo the planned exercise interventions, deferring exercise sessions or reducing exercise intensity when needed.

\section{Discussion}

The TARGET-EFT trial is testing a practical approach to identify and treat frailty in the acute cardiac setting. Identification of frailty follows a two-step process starting with the brief EFT scale, and in selected cases, additional assessments of strength, cognition, nutrition, and anemia. Both the EFT and additional assessments are easy to perform at the patient's bedside, and the tiered approach helps to more efficiently allocate resources in busy hospital environments. Treatment of frailty is multifaceted and individualized to target the patient's physical and non-physical deficits (5); aiming to mitigate the impact of hospitalization-related stressors and minimize the risk of deconditioning and post-hospitalization syndrome.

Using the EFT as the starting point has several advantages. First, feasibility, the EFT is simple, rapid to perform and does not require special equipment or lengthy questionnaires, which would likely be prohibitive in constrained clinical workflows. Second, interpretability, the four components of the EFT are each associated with specific domains (physical function, cognitive function, nutrition, anemia) that have actionable pathways for further assessment and management, unlike certain other frailty scales that output aggregated global estimates of risk that are difficult to distill. Third, prognostic value, previous studies have shown that high EFT scores signify a high-risk subpopulation of older adults that have greater risks of mortality and worsening disability and thus stand to benefit from interventions $(11,28)$.

Interventions to de-frail older adults have been previously reviewed $(5,29)$, and can be categorized into one or more of the following categories: exercise interventions, nutritional interventions, cognitive interventions (including delirium prevention programs), psychosocial interventions, and pharmacological interventions. Most of the clinical trials in this field of research have been conducted in community-dwelling older adults, with few trials addressing hospitalized patients, and even fewer trials addressing acute cardiac patients (30-34). It is this under-represented patient population that the TARGETEFT trial is seeking to include and treat. In doing so, the TARGET-EFT trial will address an important knowledge gap about the potential benefits of treating patients' frailty deficits in parallel with their cardiac deficits.

The components chosen for our intervention are supported by high-quality evidence that has shown success in treating frailty. Moreover, they are intended to be practical and amenable to real-world implementation within the confines of a hospital ward. A key requirement for implementation is to minimize extra workload for physicians, nurses, physiotherapists, and dieticians; to this end, the intervention is designed to be predominantly driven by exercise providers who will liaise with the treating team to provide and receive updates about the patients' progress and seek expertise when 
needed. While not part of our protocol, future implementations may leverage trained volunteers to help mobilize, stimulate, and feed patients, akin to the highly-successful Hospital Elder Life Program (HELP) (16).

\section{Evidence for the exercise component}

A clinical trial by Martínez-Velilla et al. showed that basic resistance exercises administered to older patients on acute geriatrics wards improved functional status, independence in ADLs, and HRQOL at discharge (35). In addition, clinical studies showed that early mobilization initiatives on acute care wards and intensive care units improved functional status (36, 37 ) and that these benefits extended to frail patients (38). Of note, a systematic review found inconsistencies in the effect of exercise interventions on frailty parameters owing to low sample sizes and lack of standardization in exercise intensity and frequency between studies (39), and less is known about the capabilities of acute cardiac patients to perform such exercise interventions.

\section{Evidence for the nutrition component}

The NOURISH clinical trial showed that a protein-rich oral nutritional supplement beverage (the same as used in the TARGET-EFT trial) administered to malnourished hospitalized older adults reduced all-cause mortality at 90 days $(40,41)$. In a similar patient population, a clinical trial showed that oral nutritional supplementation based on the MedPass protocol (also used in the TARGET-EFT trial) improved HRQOL. A meta-analysis concluded that nutritional support reduced mortality and readmissions compared to standard care in frail and pre-frail hospitalized older adults (42). Our prior research substantiated the co-prevalence of malnutrition and frailty and impact on adverse events $(43,44)$.

\section{Evidence for the cognitive component}

Given that older adults with cognitive impairment are at substantially greater risk of delirium when hospitalized, and in turn, delirium is consistently tied to downstream mortality and major morbidity (45-47), this will be the focus of our cognitive component. A meta-analysis concluded that delirium prevention programs, diverse in their respective protocols, succeeded in reducing the incidence of delirium in older patients undergoing elective surgeries (48). Also in the postoperative setting, the HELP (similar to the cognitive component of the TARGETEFT trial) reduced length of stay and improved physical and cognitive functioning in vulnerable older patients (49).

\section{Evidence for the anemia component}

There is a well-documented association between anemia and frailty, both highly prevalent in older adults with CVD, as shown in cross-sectional and longitudinal studies (50). Intravenous iron therapy may mitigate frailty as it is associated with improved HRQOL, energy level, and functional capacity (as measured by the 6-minute walk test) $(51,52)$. A meta- analysis of four clinical trials concluded that intravenous iron therapy reduced the composite endpoint of cardiovascular death or hospitalization in chronic heart failure, and the recently reported AFFIRM-AHF trial showed similar benefits in the context of acute hospitalized heart failure $(53,54)$. While deemed safe and more efficacious than oral iron for older adults, intravenous iron remains underutilized by clinicians (55).

\section{Conclusions}

To our knowledge, TARGET-EFT will be the first randomized clinical trial to test a multicomponent intervention - addressing physical and cognitive impairments as well as nutrition and anemia - to de-frail older adults hospitalized with CVD. The overarching goal is to treat patients' frailty in parallel with their CVD, and in doing so, optimize their functional losses while in-hospital and in the short-term. The results of this trial will inform best practices for patient-centered care and frailty management in this vulnerable group of patients. Importantly, the proposed assessment and intervention paradigm is amenable to translation in clinical practice.

Author contributions: RF was responsible for the conceptualization, methodology, data collection, project administration and writing of the original draft. HM was responsible for the conceptualization, methodology and data collection. FA was responsible for project administration and data collection. MG was responsible for the conceptualization and supervision. JA was responsible for the conceptualization and methodology, formal analysis, and supervision. All authors read and reviewed the final manuscript.

Acknowledgments: The authors would like to acknowledge the doctors, nurses, allied health professionals, and clerical staff at the Jewish General Hospital Azrieli Heart Centre for supporting this trial. We would like to thank Nilmini Mendis for proofreading the manuscript.

Funding: RF is supported by the Gordon Phillips Fellowship and the Graduate Excellence Fellowship from the McGill University Faculty of Medicine and Health Sciences. HM was supported through a grant provided by the Fonds de Recherche du Québec Santé (FRQS). FA is supported by the Canadian Institutes of Health Research (CIHR) Canada Graduate Scholarship.

Disclosures: None.

Conflicts of interest: none to disclose

\section{References}

1. Afilalo J, Karunananthan S, Eisenberg MJ, Alexander KP, Bergman H. Role of frailty in patients with cardiovascular disease. The American journal of cardiology. 2009;103(11):1616-21.

2. Afilalo J, Alexander KP, Mack MJ, Maurer MS, Green P, Allen LA, et al. Frailty assessment in the cardiovascular care of older adults. Journal of the American College of Cardiology. 2014;63(8):747-62.

3. Afilalo J, Lauck S, Kim DH, Lefèvre T, Piazza N, Lachapelle K, et al. Frailty in older adults undergoing aortic valve replacement: the FRAILTY-AVR study. Journal of the American College of Cardiology. 2017;70(6):689-700.

4. Solomon J, Moss E, Morin JF, Langlois Y, Cecere R, de Varennes B, et al. The Essential Frailty Toolset in Older Adults Undergoing Coronary Artery Bypass Surgery. J Am Heart Assoc. 2021;10(15):e020219.

5. Bibas L, Levi M, Bendayan M, Mullie L, Forman DE, Afilalo J. Therapeutic interventions for frail elderly patients: part I. Published randomized trials. Progress in cardiovascular diseases. 2014;57(2):134-43.

6. Krumholz HM. Post-hospital syndrome-a condition of generalized risk. The New England journal of medicine. 2013;368(2):100.

7. Wenger NK. Current status of cardiac rehabilitation. Journal of the American College of Cardiology. 2008;51(17):1619-31.

8. Covinsky KE, Palmer RM, Fortinsky RH, Counsell SR, Stewart AL, Kresevic D, et al. Loss of independence in activities of daily living in older adults hospitalized with medical illnesses: increased vulnerability with age. J Am Geriatr Soc. 2003;51(4):451-8.

9. Covinsky KE, Pierluissi E, Johnston CB. Hospitalization-associated disability: "She was probably able to ambulate, but I'm not sure". Jama. 2011;306(16):1782-93. 
10. Boyd CM, Landefeld CS, Counsell SR, Palmer RM, Fortinsky RH, Kresevic D, et al. Recovery of activities of daily living in older adults after hospitalization for acute medical illness. J Am Geriatr Soc. 2008;56(12):2171-9.

11. Bibas L, Levi M, Bendayan M, Mullie L, Forman DE, Afilalo J. Therapeutic interventions for frail elderly patients: part I. Published randomized trials. Prog Cardiovasc Dis. 2014;57(2):134-43.

12. Guralnik JM, Simonsick EM, Ferrucci L, Glynn RJ, Berkman LF, Blazer DG, et al. A short physical performance battery assessing lower extremity function: association with self-reported disability and prediction of mortality and nursing home admission. J Gerontol. 1994;49(2):M85-94.

13. Wischmeyer PE, Carli F, Evans DC, Guilbert S, Kozar R, Pryor A, et al. American Society for Enhanced Recovery and Perioperative Quality Initiative Joint Consensus Statement on Nutrition Screening and Therapy Within a Surgical Enhanced Recovery Pathway. Anesthesia \& Analgesia. 2018;126(6).

14. Folstein MF, Folstein SE, McHugh PR. "Mini-mental state": a practical method for grading the cognitive state of patients for the clinician. Journal of psychiatric research. 1975;12(3):189-98.

15. Vivifrail Project [Available from: http://vivifrail.com.

16. Inouye SK, Bogardus JS, Baker DI, Leo-Summers L, Cooney JL. The Hospital Elder Life Program: a model of care to prevent cognitive and functional decline in older hospitalized patients. Hospital Elder Life Program. Journal of the American Geriatrics Society. 2000;48(12):1697-706.

17. Campbell KL, Webb L, Vivanti A, Varghese P, Ferguson M. Comparison of three interventions in the treatment of malnutrition in hospitalised older adults: A clinical trial. Nutrition \& Dietetics. 2013;70(4):325-31

18. CADTH Rapid Response Reports. The Medication Pass Nutritional Supplement Program in Patients Receiving Medication: A Review of Clinical Effectiveness and Guidelines. Ottawa (ON): Canadian Agency for Drugs and Technologies in Health

Copyright (C) 2015 Canadian Agency for Drugs and Technologies in Health.; 2015.

19. Ezekowitz JA, O'Meara E, McDonald MA, Abrams H, Chan M, Ducharme A, et al 2017 Comprehensive Update of the Canadian Cardiovascular Society Guidelines for the Management of Heart Failure. Can J Cardiol. 2017;33(11):1342-433.

20. Price E, Artz AS, Barnhart H, Sapp S, Chelune G, Ershler WB, et al. A prospective randomized wait list control trial of intravenous iron sucrose in older adults with unexplained anemia and serum ferritin 20-200 ng/mL. Blood Cells Mol Dis 2014;53(4):221-30

21. Herdman M, Gudex C, Lloyd A, Janssen M, Kind P, Parkin D, et al. Development and preliminary testing of the new five-level version of EQ-5D (EQ-5D-5L). Quality of Life Research. 2011;20(10):1727-36.

22. Xie F, Pullenayegum E, Gaebel K, Bansback N, Bryan S, Ohinmaa A, et al. A Time Trade-off-derived Value Set of the EQ-5D-5L for Canada. Med Care. 2016;54(1):98105 .

23. Fillenbaum GG, Smyer MA. The Development, Validity, and Reliability of the Oars Multidimensional Functional Assessment Questionnaire. Journal of Gerontology. 1981;36(4):428-34

24. Malmstrom TK, Miller DK, Simonsick EM, Ferrucci L, Morley JE. SARC-F: a symptom score to predict persons with sarcopenia at risk for poor functional outcomes. J Cachexia Sarcopenia Muscle. 2016;7(1):28-36.

25. Zigmond AS, Snaith RP. The hospital anxiety and depression scale. Acta Psychiatr Scand. 1983;67(6):361-70.

26. McClure NS, Sayah FA, Xie F, Luo N, Johnson JA. Instrument-Defined Estimates of the Minimally Important Difference for EQ-5D-5L Index Scores. Value Health. 2017;20(4):644-50

27. Borg GA. Psychophysical bases of perceived exertion. Med Sci Sports Exerc 1982;14(5):377-81.

28. Theou O, Squires E, Mallery K, Lee JS, Fay S, Goldstein J, et al. What do we know about frailty in the acute care setting? A scoping review. BMC geriatrics. 2018;18(1):120 .

29. Bendayan M, Bibas L, Levi M, Mullie L, Forman DE, Afilalo J. Therapeutic interventions for frail elderly patients: part II. Ongoing and unpublished randomized trials. Prog Cardiovasc Dis. 2014;57(2):144-51.

30. Apóstolo J, Cooke R, Bobrowicz-Campos E, Santana S, Marcucci M, Cano A. et al. Effectiveness of interventions to prevent pre-frailty and frailty progression in older adults: a systematic review. JBI Database System Rev Implement Rep. 2018;16(1):140-232.

31. Travers J, Romero-Ortuno R, Bailey J, Cooney MT. Delaying and reversing frailty: a systematic review of primary care interventions. Br J Gen Pract. 2019;69(678):e61-e9.

32. Negm AM, Kennedy CC, Thabane L, Veroniki AA, Adachi JD, Richardson J, et al. Management of Frailty: A Systematic Review and Network Meta-analysis of Randomized Controlled Trials. J Am Med Dir Assoc. 2019;20(10):1190-8

33. Rezaei-Shahsavarloo Z, Atashzadeh-Shoorideh F, Gobbens RJJ, Ebadi A, Ghaedamin Harouni G. The impact of interventions on management of frailty in hospitalized frail older adults: a systematic review and meta-analysis. BMC Geriatrics. 2020;20(1):526.

34. Ng TP, Feng L, Nyunt MS, Feng L, Niti M, Tan BY, et al. Nutritional, Physical, Cognitive, and Combination Interventions and Frailty Reversal Among Older Adults: A Randomized Controlled Trial. Am J Med. 2015;128(11):1225-36.e1.

35. Martínez-Velilla N, Casas-Herrero A, Zambom-Ferraresi F, Sáez de Asteasu ML,
Lucia A, Galbete A, et al. Effect of Exercise Intervention on Functional Decline in Very Elderly Patients During Acute Hospitalization. JAMA Internal Medicine. 2019;179(1).

36. Schweickert WD, Pohlman MC, Pohlman AS, Nigos C, Pawlik AJ, Esbrook CL, et al. Early physical and occupational therapy in mechanically ventilated, critically ill patients: a randomised controlled trial. Lancet. 2009;373(9678):1874-82.

37. Schaller SJ, Anstey M, Blobner M, Edrich T, Grabitz SD, Gradwohl-Matis I, et al. Early, goal-directed mobilisation in the surgical intensive care unit: a randomised controlled trial. Lancet. 2016;388(10052):1377-88

38. Goldfarb M, Afilalo J, Chan A, Herscovici R, Cercek B. Early mobility in frail an non-frail older adults admitted to the cardiovascular intensive care unit. J Crit Care. 2018;47:9-14.

39. Scheerman K, Raaijmakers K, Otten RHJ, Meskers CGM, Maier AB. Effect of physical interventions on physical performance and physical activity in older patients during hospitalization: a systematic review. BMC Geriatrics. 2018;18(1):288.

40. Deutz NE, Matheson EM, Matarese LE, Luo M, Baggs GE, Nelson JL, et al. Readmission and mortality in malnourished, older, hospitalized adults treated with a specialized oral nutritional supplement: A randomized clinical trial. Clin Nutr 2016;35(1):18-26.

41. Matheson EM, Nelson JL, Baggs GE, Luo M, Deutz NE. Specialized oral nutritional supplement (ONS) improves handgrip strength in hospitalized, malnourished older patients with cardiovascular and pulmonary disease: A randomized clinical trial. Clin Nutr. 2020.

42. Gomes F, Baumgartner A, Bounoure L, Bally M, Deutz NE, Greenwald JL, et al Association of Nutritional Support With Clinical Outcomes Among Medical Inpatients Who Are Malnourished or at Nutritional Risk: An Updated Systematic Review and Meta-analysis. JAMA Netw Open. 2019;2(11):e1915138.

43. Goldfarb M, Lauck S, Webb JG, Asgar AW, Perrault LP, Piazza N, et al. Malnutrition and Mortality in Frail and Non-Frail Older Adults Undergoing Aortic Valve Replacement. Circulation. 2018;138(20):2202-11.

44. Goldfarb M, Marcano Y, Schafer D, Chronopoulos J, Hayman V, Trnkus A, et al. Dietary protein intake in older adults undergoing cardiac surgery. Nutrition, Metabolism and Cardiovascular Diseases. 2019;29(10):1095-100.

45. Noriega FJ, Vidán MT, Sánchez E, Díaz A, Serra-Rexach JA, Fernández-Avilés $\mathrm{F}$, et al. Incidence and impact of delirium on clinical and functional outcomes in older patients hospitalized for acute cardiac diseases. American Heart Journal 2015;170(5):938-44.

46. Ibrahim K, McCarthy CP, McCarthy KJ, Brown CH, Needham DM, Januzzi Jr JL, et al. Delirium in the cardiac intensive care unit. Journal of the American Heart Association. 2018;7(4):e008568.

47. Vidán MT, Sánchez E, Alonso M, Montero B, Ortiz J, Serra JA. An intervention integrated into daily clinical practice reduces the incidence of delirium during hospitalization in elderly patients. Journal of the American Geriatrics Society. 2009;57(11):2029-36.

48. Janssen TL, Alberts AR, Hooft L, Mattace-Raso F, Mosk CA, van der Laan L. Prevention of postoperative delirium in elderly patients planned for elective surgery: systematic review and meta-analysis. Clinical interventions in aging. 2019;14:1095117.

49. Wang YY, Yue JR, Xie DM, Carter P, Li QL, Gartaganis SL, et al. Effect of the Tailored, Family-Involved Hospital Elder Life Program on Postoperative Delirium and Function in Older Adults: A Randomized Clinical Trial. JAMA Intern Med. 2019.

50. Palmer K, Vetrano DL, Marengoni A, Tummolo AM, Villani ER, Acampora N, et al. The Relationship Between Anaemia and Frailty: A Systematic Review and Meta-Analysis of Observational Studies. The journal of nutrition, health \& aging 2018;22(8):965-74.

51. Qian C, Wei B, Ding J, Wu H, Wang Y. The Efficacy and Safety of Iron Supplementation in Patients With Heart Failure and Iron Deficiency: A Systematic Review and Meta-analysis. Canadian Journal of Cardiology. 2016;32(2):151-9.

52. Avni T, Leibovici L, Gafter-Gvili A. Iron supplementation for the treatment of chronic heart failure and iron deficiency: systematic review and meta-analysis. Eur J Heart Fail. 2012;14(4):423-9.

53. Anker SD, Kirwan BA, van Veldhuisen DJ, Filippatos G, Comin-Colet J, Ruschitzka $\mathrm{F}$, et al. Effects of ferric carboxymaltose on hospitalisations and mortality rates in irondeficient heart failure patients: an individual patient data meta-analysis. Eur J Heart Fail. 2018;20(1):125-33.

54. Ponikowski P, Kirwan BA, Anker SD, McDonagh T, Dorobantu M, Drozdz J, et al. Ferric carboxymaltose for iron deficiency at discharge after acute heart failure: a multicentre, double-blind, randomised, controlled trial. Lancet 2020;396(10266):1895-904

55. Auerbach M, Spivak J. Treatment of Iron Deficiency in the Elderly: A New Paradigm. Clinics in Geriatric Medicine. 2019;35(3):307-17.

How to cite this article: Fountotos, R., Munir, H., Ahmad, F. et al. Rationale and Design of the TARGET-EFT Trial: Multicomponent Intervention for Frailt and Pre-frail Patients Hospitalized with Acute Cardiac Conditions. J Nutr Health Aging. 2022;26(3):282-289, https://doi.org/10.1007/s12603-022-1759-y 